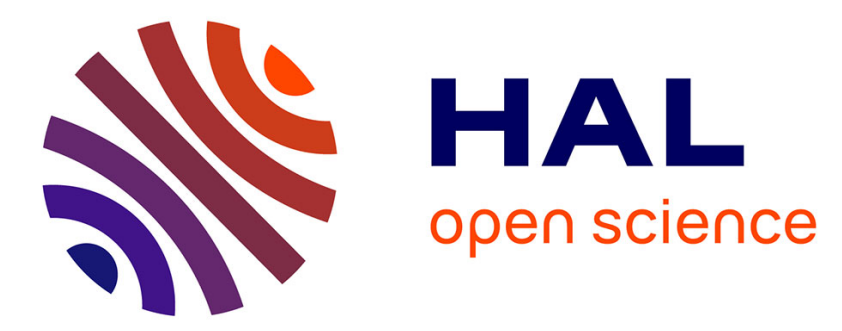

\title{
Évaluation des effets des zonages environnementaux sur la croissance urbaine et l'activité agricole
}

\author{
Ghislain Geniaux, Claude Napoleone
}

\section{To cite this version:}

Ghislain Geniaux, Claude Napoleone. Évaluation des effets des zonages environnementaux sur la croissance urbaine et l'activité agricole. Economie et Statistique / Economics and Statistics, 2011, 444-445, pp.181-199. hal-01369343

\section{HAL Id: hal-01369343 \\ https://hal.science/hal-01369343}

Submitted on 28 May 2020

HAL is a multi-disciplinary open access archive for the deposit and dissemination of scientific research documents, whether they are published or not. The documents may come from teaching and research institutions in France or abroad, or from public or private research centers.
L'archive ouverte pluridisciplinaire HAL, est destinée au dépôt et à la diffusion de documents scientifiques de niveau recherche, publiés ou non, émanant des établissements d'enseignement et de recherche français ou étrangers, des laboratoires publics ou privés. 


\title{
Évaluation des effets des zonages environnementaux sur la croissance urbaine et l'activité agricole
}

\author{
Ghislain Geniaux et Claude Napoléone *
}

\begin{abstract}
La promulgation de nouvelles zones de protection de l'environnement dans les régions les plus soumises à la pression urbaine se heurte quelquefois à des réticences : on leur reproche de réduire l'offre foncière et, par là-même, de nuire au développement économique et social du reste de la commune. Or, la préservation des milieux naturels nécessite de densifier les zones déjà urbanisées et de limiter l'extension de nouvelles zones constructibles.
\end{abstract}

Une évaluation portant sur la région Provence-Alpes-Côte d'Azur et utilisant une méthode d'expériences quasi-naturelles (techniques de double différence avec appariement) montre que la croissance du nombre de logements a été plus soutenue dans les communes comportant une zone environnementale stricte que dans des communes similaires n'ayant pas accueillies de telles zones. De plus, on n'y observe pas pour autant une baisse de la population agricole. Se trouve ainsi infirmé l'argument selon lequel les zonages environnementaux seraient un frein à la croissance urbaine et au maintien de l'activité agricole.

À l'échelon infra-communal, la construction a été sensiblement freinée au voisinage immédiat des zonages environnementaux stricts, comparativement aux autres zones naturelles de la commune. Dans cette région, ces zones exercent donc une protection effective des milieux naturels. Cela conduit à avancer que les zonages environnementaux participent à l'émergence d'aménités contribuant à rendre attractive leur commune d'accueil, tout en maintenant un objectif de protection des milieux naturels et de densification urbaine. 
$\mathbf{L}$ es zonages ont été utilisés depuis plus d'un siècle par les pays développés pour protéger un certain nombre d'espèces ou d'espaces naturels par l'exclusion des activités perturbatrices (Deverre et de Sainte Marie, 2008). Or, dans les régions soumises à une forte croissance urbaine, la promulgation de nouveaux zonages environnementaux est rendue difficile car ils sont considérés comme des dispositifs figeant ou perturbant l'évolution des territoires. Un argument important est que la restriction de l'offre inhérente à la protection d'une partie du territoire est susceptible de faire croître les prix fonciers sur le reste des espaces constructibles et agricoles. Ils perturberaient par-là même l'accès au logement pour les ménages les plus modestes et à la terre pour l'agriculture.

À notre sens, la nature de la relation entre promulgation d'un zonage environnemental et activité de construction ou agricole reste à vérifier. Dans cette perspective, notre analyse vise à déterminer si les zonages environnementaux ont un effet sur la croissance urbaine et si, directement ou indirectement, ils ont également un effet sur la population agricole. Dès lors qu'ils ont comme objectif explicite de contenir l'urbanisation en leur sein, nous les dénommerons « zonages stricts ». Leur performance peut tout d'abord être évaluée en considérant le nombre de bâtiments qui s'y sont érigés depuis leur création. Toutefois, la performance interne des zonages environnementaux est également susceptible d'induire une pression d'urbanisation plus importante dans leur voisinage :

- D’abord par un effet mécanique de diminution de l'offre foncière. Les bâtiments qui auraient pu se localiser dans les zones protégées se reportent dans les zones pouvant accueillir l'urbanisation. Ce report peut être jugé bénéfique au regard de la préservation des espaces naturels, lorsqu'il se fait vers les zones d'urbanisation existantes. Il peut être jugé négatif s'il conduit à une urbanisation des autres espaces naturels ou agricoles existants à proximité.

- Ensuite par un effet d'attraction : de nouvelles constructions peuvent se localiser à proximité de la zone protégée afin de bénéficier des externalités paysagères qu'elle délivre (Towe et Bockstael, 2005).

Pour évaluer les effets de la promulgation de zonages environnementaux stricts sur la croissance urbaine et l'activité agricole, nous avons réalisé une analyse ex post des conséquences de leur mise en place sur l'évolution du nombre de logements ainsi que du nombre d'actifs et d'exploitations agricoles, au sein des communes de la région Provence-Alpes-Côte d'Azur (Paca). Nous avons conduit notre évaluation à partir d'expériences quasi-naturelles (EQN) utilisant des techniques de double différence avec appariement (1) (Imbens et Wooldridge 2009 ; Heckman et al., 1998). Ces méthodes permettent d'estimer les effets propres des politiques étudiées. Elles seront mises en œuvre en distinguant les échelles communales et infra-communales. À l'échelle communale, nous comparons les évolutions des logements résidentiels et de la population agricole entre communes dotées de zonages environnementaux et d'autres n'en disposant pas. À l'échelle infra-communale, nous nous intéressons aux effets de voisinage des zonages environnementaux sur les densités résidentielles.

\section{État de l'art}

En économie, les effets des zonages ont principalement été étudiés en les replaçant dans deux perspectives :

- Les effets sur les équilibres financiers et démographiques de la commune, au titre de l'analyse des déterminants d'adoption. Le zonage est alors considéré comme le moyen d'influer sur l'agencement des usages du sol (au titre du paysage : McDonald et McMillen, 2004), sur le potentiel fiscal local (via le nombre de ménages : McDonald et McMillen, 2004) et sur la structure la structure sociodémographique des communes (sélection de groupes sociaux : Stigler, 1971 ; Ellickson, 1977 ; Fischel, 1985).

- Les effets sur les prix des biens fonciers et immobiliers et sur le volume de l'offre foncière, avec comme problématique de fond la légitimité de ce type d'outils. Jaeger et Plantinga (2007) identifient par exemple trois types d'effets par lesquels le zonage influence les prix : un effet de restriction (Restriction effects) qui, en limitant les usages possibles d'une parcelle, réduit son prix par rapport à son utilisation la plus rentable ; un effet d'aménité (Amenity effects) qui par la séparation spatiale des usages du sol permet d'une part d'éviter certaines nuisances (le bruit ou la pollution industrielle) et d'autre part de générer des externalités positives de

\footnotetext{
1. Les méthodes d'appariement concernées relèvent du Propensity Score Matching (PSM) proposés par Rosenbaum et Dubin (1983) visant à réduire le biais de sélection - voir encadré 1 pour plus de détails.
} 
voisinage en rendant la proximité des résidences plus plaisante et plus durable ; un effet de rareté (Scarcicty effects) résultant de l'évolution de l'offre potentielle au regard de la demande foncière et immobilière.

Toutefois, l'évaluation empirique des effets de la promulgation d'un zonage présente des difficultés tenant à la présence de biais de sélection. En effet, le nombre de communes où l'usage du sol n'est pas zoné, à un titre ou un autre, est très faible (Evans 1999). Il est donc impossible de disposer d'un nombre suffisant de situations observables où un zonage strict se met en œuvre sur un espace vierge de régulation. Pour contourner cette difficulté, une stratégie possible consiste à s'intéresser à des actions zonales nouvelles qui se surajoutent au contexte existant. Dans cette perspective, la méthode des EQN, conjointement à l'utilisation de systèmes d'observation performants des politiques d'urbanisme et environnementales (numérisation et système d'information géographique (SIG)) ont ouvert de nouveaux horizons. S'inspirant des méthodes d'expériences naturelles utilisées en médecine, les EQN construisent un dispositif d'observation visant à comparer les évolutions avant et après traitement sur un groupe « traité » (les individus bénéficiant de l'action publique étudiée) et un groupe « de contrôle » (des individus similaires non soumis à la même action publique). On se reportera à Imbens et Wooldrige (2008) pour une revue détaillée de ce type de méthode et à Fougère (2010) pour une revue en langue française. La similarité des situations initiales entre le groupe traité et le groupe de contrôle permet de contrôler les biais de sélection et l'observation conjointe des évolutions des deux situations permet d'attribuer les changements observés à la nouvelle action publique qui se sur-rajoute aux contextes initiaux. On mentionnera Zhou, McMillen et McDonnald (2008), Anderson et Weinhold (2008), Lynch et Liu (2007), Lynch et al. (2007) et Netusil (2005). Comparant l'urbanisation entre deux périodes dans le Maryland, Towe et Bockstael (2005) montrent par exemple que l'urbanisation des parcelles contiguës à celles ayant fait l'objet d'une mesure de conservation (Conservation Easement) est plus intense que sur le reste de la zone observée (2). C'est dans la continuité méthodologique de ces articles que nous plaçons notre contribution. Nous y introduisons la question de l'évaluation des effets directs et indirects des zonages environnementaux stricts au sein de la commune où ils sont établis et dans leur voisinage immédiat et nous nous focalisons sur le logement résidentiel et sur l'activité agricole.

\section{Trois classes de zonages environnementaux}

Un mode important de préservation des milieux naturels est l'action publique zonale (cf. encadré 2). Elle s'inscrit dans une politique « d'aires protégées » déjà ancienne qui nous vaut de dénombrer aujourd'hui une grande variété de zonages environnementaux (environ une trentaine). Ces zonages se recouvrent souvent, ce qui conduit sur les espaces concernés à un empilement de mesures réglementaires. Or, les conséquences pratiques sur l'urbanisme des zonages

2. Les espaces naturels y sont considérés comme des caractéristiques de voisinage recherchées pour les nouvelles habitations et leur protection rassure promoteurs, acheteurs ou constructeurs sur la durabilité des caractéristiques de l'environnement.

Encadré 1

\section{ESTIMATION DU SCORE DE PROPENSION}

Nous utilisons un modèle Logit non-paramétrique géoadditif (Wood 2006) appartenant à la famille des modèles additifs généralisés (GAM) (Hastie et Tibshirani 1990). Ces modèles sont une extension des modèles linéaires généralisés où les prédicteurs sont spécifiés sous la forme d'une somme de fonctions lissées des régresseurs. Les fonctions lissées utilisées dans notre cas sont des spline et le modèle est estimé à partir d'un maximum de vraisemblance pénalisé (P-IRLS pour " penalized iteratively reweighted least square ", avec un paramètre de lissage qui équilibre le taux de lissage et la qualité de la prédiction). Le modèle prend la forme suivante:

$P_{(z)}=s\left(z_{1}\right)+s\left(z_{2}\right)+\ldots+s\left(z_{k}\right)+s(x, y)+\varepsilon$ où les variables $z_{1}$ à $z_{k}$ sont des variables environnementales, démographiques et économiques jouant sur la probabilité d'avoir un zonage environnemental strict (Zest) sur la commune. Lorsque l'on intègre également la longitude et la latitude du barycentre de la commune $(x, y)$, afin de corriger des effets des variables omises et de limiter l'hétérogénéité spatiale, le modèle est dit GAM géoadditif. II ne se distingue des modèles GAM classiques que de ce point de vue (voir Geniaux et Napoléone (2008) pour une introduction au modèle géoadditif). Le modèle GAM géoadditif utilisé est par ailleurs un modèle logit puisque l'endogène (présence/ absence d'un Zest) est de nature binaire. 
existants sont très diverses. Elles vont de procédures non explicitement exclusives de l'urbanisation (les inventaires Znieff par exemple (3)), aux dispositifs opposables au tiers, rédhibitoires à toute urbanisation nouvelle (zone centrale des parcs, réserve naturelle nationale, etc.). De plus, en corollaire des dispositions légales statuant sur la constructibilité au sein d'un zonage, il existe une grande variété de pratiques institutionnelles qui sont susceptibles de leur conférer une action particulière sur la localisation des nouvelles zones constructibles. À titre d'exemple, selon la sensibilité des élus locaux à l'environnement, une Znieff peut ne pas avoir d'effet sur un nouveau document d'urbanisme comme elle peut influer sur la localisation des espaces naturels et urbains de la commune. Ces éléments d'appréciation locale ne peuvent être évalués que par des travaux de terrain réalisés auprès des acteurs en charge de leur mise en œuvre (en l'occurrence les communes et les intercommunalités promulguant des documents d'urbanisme d'un coté et les services déconcentrés de l'État d'un autre, au titre du contrôle de légalité). Dans la perspective de caractériser les différents zonages existant en Paca, eu égard à leur prise en compte dans les documents d'urbanisme, nous avons constitué et animé un comité d'experts de 2003 à 2008, composé d'une quinzaine de représentants des services déconcentrés de l'État (agents de la
Direction régionale de l'environnement, de l'aménagement et du logement (DREAL) en charge de l'urbanisme et de l'environnement), de techniciens d'agences d'urbanisme, de représentant de collectivités territoriales (de la commune à la région). Ce comité a été mis en place dans le cadre de la construction d'un modèle de simulation de l'urbanisation (URBASNISMUL). Les propositions du groupe d'experts ont été validées par confrontations aux documents d'urbanisme existants (dont nous disposons des contours) et par enquêtes auprès d'élus locaux. Il en résulte une typologie en trois classes de zonages environnementaux considérés comme homogènes $\mathrm{du}$ point de vue de l'inconstructibilité : les zonages stricts (Zest), les zonages souples (Zesp) et les zonages de connaissance (Zeco).

- Dans les zonages stricts, il est interdit de construire et inenvisageable de rendre la zone constructible dans les POS/PLU (4). Ils sont composés de six dispositifs : les arrêtés de bio-

\footnotetext{
3. Zone naturelle d'intérêt écologique, faunistique et floristique. 4. Le plan d'occupation des sols (POS) et le plan local d'urbanisme (PLU) sont des documents cartographiques rendant compte des zones sur lesquelles repose la planification des usages du sol sur une commune. Les documents promulgués avant le 13 décembre 2000 (Loi Solidarité et Renouvellement Urbain) sont des POS, après cette date ils ont été remplacés par les PLU.
}

\section{Encadré 2}

\section{LES POLITIQUES ZONALES DE PROTECTION DES MILIEUX NATURELS}

Le zonage constitue en France, dès le début du $X X$ siècle, l'outil central des politiques de protection des milieux naturels, avec l'instauration d'aires protégées où les activités de l'homme sont exclues ou très largement restreintes (réserves et sites remarquables : 1930, Parc nationaux : 1960). On dénombre actuellement plus d'une quinzaine de zonages environnementaux différents provenant aussi bien de l'application de conventions internationales (Man And Biosphere, convention de Ramsar relative aux zones humides d'importance internationale), de directives européennes (directives habitats, directive oiseaux), de politiques nationales (conservatoire du littoral, parc nationaux, réserves naturelles) ou de politiques des collectivités locales (parc naturel régional). Les zonages les plus anciens ont été conçus comme des espaces d'où les activités humaines sont exclues (réserves naturelles, zones centrales des parcs nationaux). À ce titre, ils comptent au nombre des premiers zonages régulant l'urbanisation, et précèdent même parfois les documents d'urbanisme. Par la suite, certains zonages environnementaux ont eu d'autres objectifs, non exclusifs des activités humaines, notamment Iorsqu'elles contribuent à la qualité, à l'entretien ou à l'existence de certains types d'habitat (agriculture, pâturage, sylviculture, habitation temporaire, etc.). Dans cette catégorie, on distingue :

- Les inventaires naturalistes au sein desquels la connaissance scientifique du moment est synthétisée dans un document mis à la disposition du public ou des institutions de gestion. C'est le cas des zones naturelles d'intérêt écologique, faunistique et floristiques (Znieff) par exemple, qui ont permis de définir et de cartographier près de 15000 zones en France depuis 1982.

- Les documents de gestion où, outre l'inventaire naturaliste justifiant leur existence, des objectifs en termes de gestion sont fixés entre les opérateurs participant à l'opération. C'est le cas par exemple des listes biogéographiques des sites « Natura 2000 » qui prévoient des documents d'objectifs (Docob) destinés à orienter les modes d'intervention des gestionnaires du site. C'est également le cas des volets environnementaux des chartes des parcs naturels régionaux, intercommunalités de projet au sein desquelles les questions environnementales sont mises en regard des autres dynamiques sociales et économiques des communes adhérentes. 
tope, les réserves naturelles nationales, les zones centrales des parcs nationaux, les réserves de biosphères, les sites inscrits, les sites classés et les propriétés du conservatoire du littoral.

- Les zonages souples sont ceux dont l'effet sur l'inconstructibilité est plus hétérogène, dépendante de l'appréciation des prescripteurs des documents d'urbanisme. Ils concernent les zones spéciales de conservation (ZSC), les zones de protection spéciale (ZPS), les zones périphériques des parcs nationaux, les zones Ramsar (5), les réserves biologiques dirigées, les réserves de l'office national des forêts (ONF), les réserves naturelles volontaires et les zones importantes pour la conservation des oiseaux (Zico).

- Les zonages de connaissances n’ont pas, en tant que tels, d'effets spécifiques sur la constructibilité. Ils regroupent les inventaires Znieff I et II (Geniaux et al., 2009).
Supprimer les recouvrements spatiaux des zonages appartenant à une même classe fournit une première appréciation de leur importance relative et de leur répartition spatiale dans les 963 communes de la région (cf. carte 1). Les espaces entrant dans le champ de compétence du conservatoire des espaces littoraux et des rivages lacustres (CELRL) n'ont pas été prises en compte dans le présent article. En effet, la loi « littoral » permet d'avoir une action protectrice dans la bande des 100 mètres (hors noyaux villageois) dans toutes les communes littorales, et pas seulement dans les seules propriétés du CELRL.

Parallèlement aux zonages environnementaux, les documents d'urbanisme disposent de zones

5. La convention de Ramsar est un traité intergouvernemental qui sert de cadre à l'action nationale et à la coopération internationale pour la conservation et l'utilisation des zones humides et de leurs ressources - voir : http://www.ramsar.org/cda/fr/ramsar-home/ main/ramsar/1_4000_1_.

\section{Carte 1}

Les zonages environnementaux stricts (Zest)

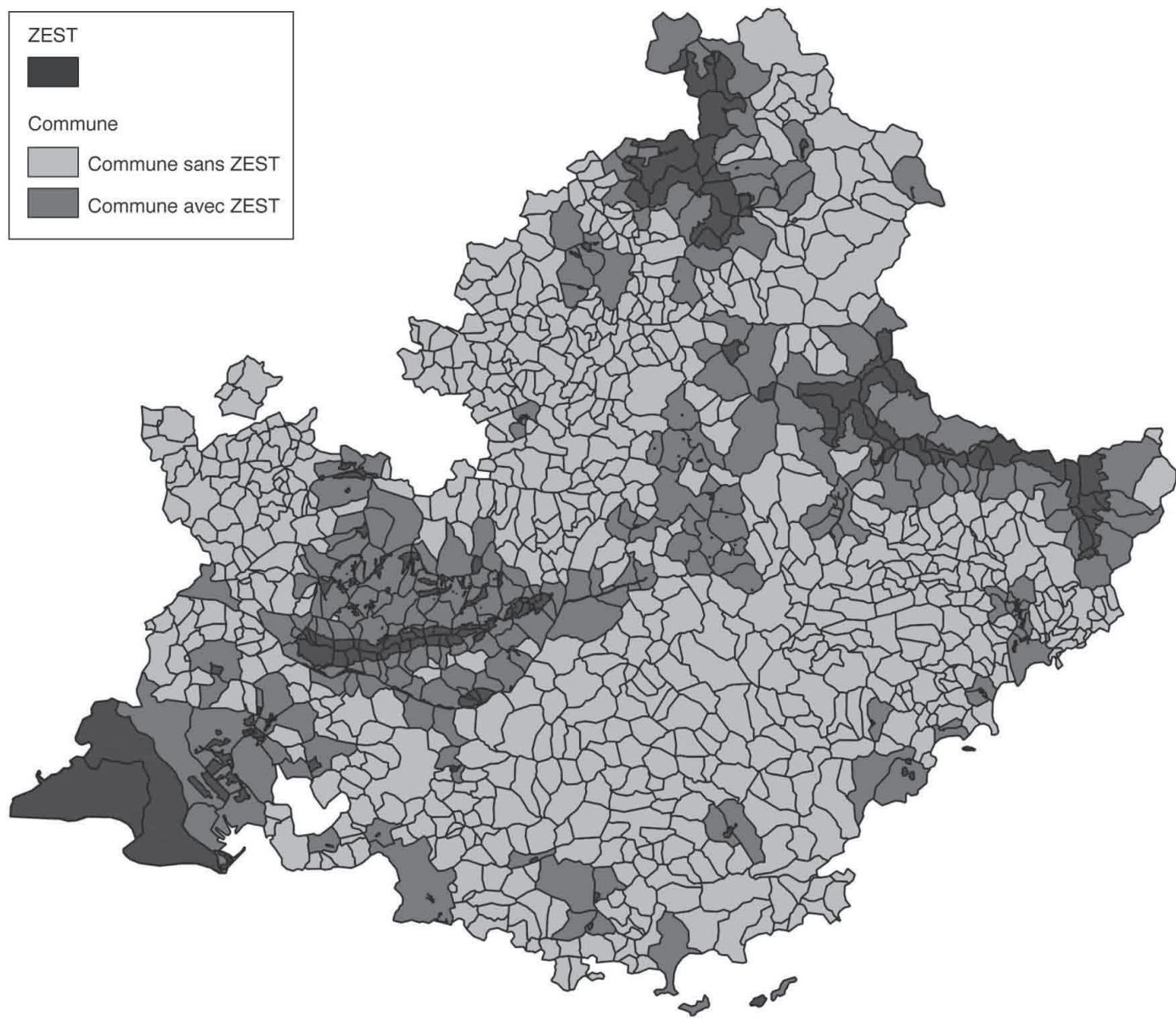

Sources : DREAL, MNHM, CRIGE PACA. 
naturelles (zones ND des plans d'occupation des sols (POS) et $\mathrm{N}$ des plans locaux d'urbanisme (PLU)) dont les niveaux de protection dépendent des règlements locaux et dont la durée est limitée à la durée de vie du document d'urbanisme lui-même. Les zones ND ou N prennent en compte l'ensemble des autres zonages environnementaux ayant un effet réglementaire sur l'urbanisation (lorsqu'une réserve naturelle nationale est promulguée, la transcription de son périmètre dans le POS/PLU se fera au sein des zones naturelles). Ils constituent donc un moyen de disposer d'une vision synthétique des différentes situations locales, eu égard à la gestion des espaces naturels. De plus, les documents d'urbanisme sont présents dans la grande majorité des communes, en dehors de villages ruraux restés aux modalités d'application du règlement national d'urbanisme. En Paca en 2006, on dénombre 852 communes sur 963 couvertes soit par un POS/PLU (754 communes), soit par une carte communale (98 communes). Seules 111 très petites communes n'ont aucun document d'urbanisme zonal : elles concernent $0,28 \%$ de la population régionale (cf. carte 2 ).

\section{Mesurer l'effet d'une politique publique : groupe traité et groupe de contrôle}

Les données relatives à l'urbanisation, aux zonages, à l'environnement et aux activités agricoles sont détaillées dans l'encadré 3. Elles sont regroupées à un niveau fin (commune ou parcelle cadastrale) dans le cadre d'un système d'information géographique (SIG) historique (données spatiales longitudinales). Elles servent à mettre en œuvre des modèles de causalité à la Rubin/Roy qui permettent de mesurer des effets propres de politiques publiques à partir de données non expérimentales. Ces méthodes visent à « reconstruire » la situation d'un échantillon d'individus statistiques soumis à un programme d'action publique dans l'hypothèse où ce programme n'aurait pas été mis en œuvre. Il est possible d'observer les effets d'un tel programme sur un individu qui en a fait l'objet. En revanche, on ne peut pas observer l'évolution qu'il aurait eue s'il n'avait pas bénéficié de ce programme. Les évolutions potentielles qui ne peuvent pas être observées sont reconstruites en identifiant un échantillon d'individus similaires

Carte 2

Les POS/PLU numérisés (Paca, 2006)

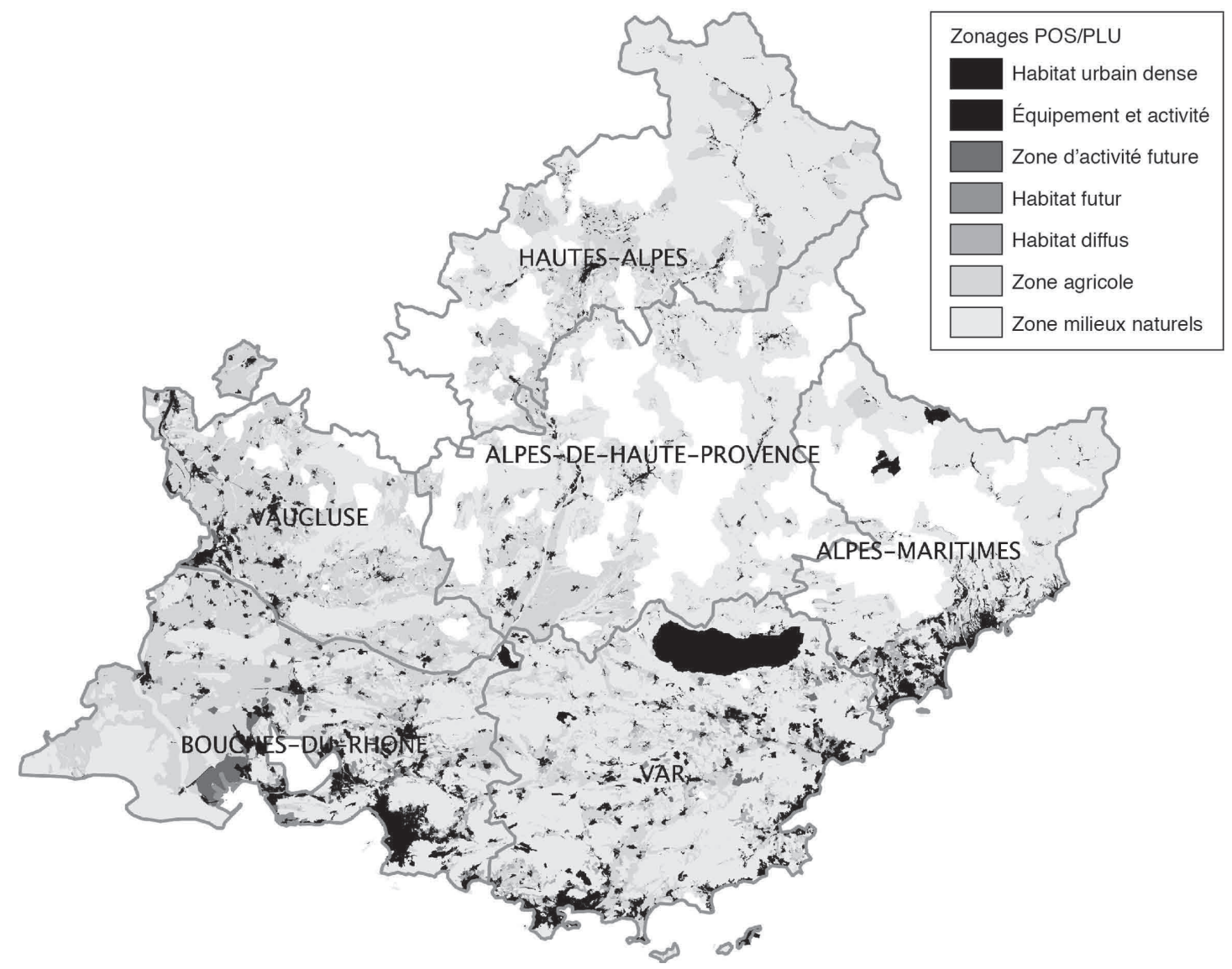

Sources : DREAL, CRIGE PACA. 
non soumis au programme d'action publique, appelé groupe de contrôle ou groupe non traité. Ces groupes de contrôle sont construits dans différents cadres d'hypothèses sur les relations entre les variables endogènes, les variables exogènes observables et non observables (6). La plupart des travaux en " sélection sur observable » s'appuient sur des appariements mis en œuvre à partir du score de propension (Rosenbaum et Rubin, 1983). Dans ce cadre d'hypothèse, la propriété d'indépendance conditionnellement à des variables observables implique celle d'indépendance conditionnellement à la probabilité de recevoir le traitement. Le passage d'un appariement sur $n$ variables à un appariement sur une seule variable (le score de propension) permet de simplifier la procédure et d'élargir le nombre de situations pour lesquelles l'appariement devient possible.

On peut également s'appuyer sur un cadre de « sélection sur inobservables » qui est moins restrictif. Ainsi, lorsque les données le permettent, un indicateur en double différence (Ashenfelter, 1978) permet de comparer les évolutions de la variable endogène entre deux périodes et entre le groupe traité et de contrôle. L'approche en double différence permet donc d'éliminer les effets fixes et les effets temporels des variables inobservables en utilisant simplement deux différences, à la manière de l'estimateur «within » en économétrie de panel. Dans la continuité des travaux de McMillen et McDonald 2002 sur l'effet propre des zonages, notre méthodologie s'appuie sur un estimateur en double différence semi-paramétrique. Nous utilisons différents modes d'appariement, notamment de type caliper (Cochran et Rubin, 1973) ou par noyau (Heckman et al 1998, Abadie 2005) largement utilisés en matière d'évaluation de politique publique (voir en annexe une description détaillée du mode d'appariement et des estimateurs).

\section{Effets mesurés et construction des individus statistiques}

L'évaluation porte sur les effets indirects de la promulgation de Zest dans un certain nombre de communes de Paca, sur la dynamique urbaine et

6. Deux hypothèses prévalent : la première est l'absence d'interaction entre observations (" stable unit treatment value assumption » ou SUTVA (Rubin, 1978). La seconde est l'indépendance conditionnelle de la variable d'intérêt $Y$ et du traitement $D$, sachant un ensemble d'observables $X$ (plus précisément $\left.D_{i} \perp\left(Y_{i}\left(D_{i}-0\right), Y_{i}\left(D_{i}-1\right)\right) \mid X\right)$.

Encadré 3

\section{LES DONNÉES}

Les données que nous avons utilisées ont fait l'objet d'un lourd travail de géomatique consistant à construire un système d'information géographique (SIG) historique (1) sur l'urbanisation et les zonages publics à l'échelle communale sur la région Paca (source DREAL/INRA) et du parcellaire cadastral sur $478 \mathrm{com}$ munes de la région pour lesquelles on dispose à la fois des cadastres et des POS/PLU numérisés (1 784200 habitants, 2583265 parcelles). Chaque bâtiment d'habitation est positionné sur sa parcelle cadastrale et renseigné par les éléments issus de la table attributaire du cadastre (première date de construction d'une parcelle, nombre de logements dans la parcelle ou un groupe de parcelles pour chaque année suivant la première construction). À partir de la date de construction, nous avons reconstruit le processus spatial d'urbanisation, renseigné en longitude et latitude, ainsi qu'en continu dans le temps, pour chaque élément bâti (614 695 parcelles ont des éléments bâtis renseignés en 2007 sur la zone d'étude). En dehors de l'ensemble des zones issues des documents d'urbanisme, le SIG historique contient les zonages environnementaux (date de prescription, type de restriction, source DREAL, MNHN), répartis selon les trois classes de zonages environnementaux décrites plus haut. Nous avons également utilisé une gamme de variables environnementales issues d'un traitement de la couche d'occupation du sol Corine land Cover
(CLC) 2005 qui détaille une cinquantaine de classes d'occupation du sol et de couverts naturels (2) et du modèle numérique de terrain (MNT) 2000 de l'institut géographique national (IGN) qui permet de décrire le relief (3). Les autres variables candidates sur la démographie, les activités agricoles ou économiques ainsi que la composition sociale des communes sont issues des recensements de la population (1962 à 1999), des recensements généraux de l'agriculture (1979-2000 - MAAPRAT (4)) et de l'inventaire communal 1998 (Insee). Enfin, des indicateurs sur les marchés fonciers sont utilisés. Ils sont issus de traitements de données sur les mutations foncières recueillies par les notaires et par la Safer (5) sur la période 2000-2006 (Voir le dossier «Observation Foncière " du $n^{\circ} 139$ de la revue Études Foncières).

\footnotetext{
1. Un système d'information géographique (SIG) est un système d'information permettant d'organiser et de présenter des données spatialement référencées. On le qualifie d'historique lorsque l'on dispose de plusieurs dates d'observation pour les attributs qui renseignent chaque objet géographique.

2. Voir : http://sd1878-2. sivit.org/.

3. Voir : http://professionnels.ign.fr/ficheProduitCMS.do?idDoc $=5323461$.

4. Ministère de l'agriculture, de l'alimentation, de la pêche et de l'aménagement du territoire.

5. Société d'aménagement foncier et d'établissement rural.
} 
agricole : cela revient à estimer les différences d'évolution entre les années 1970 et 2005, entre les unités statistiques qui ont subi les conséquences d'un classement de portions de territoire en Zest (ces unités pouvant être des communes, ou des fractions de communes (cf. annexe) ; conformément à ce qui précède elles seront dénommées 《 individus traités ») et des unités de caractéristiques comparables mais n'ayant pas fait l'objet de traitement (individus appartenant au « groupe de contrôle »). Ces évaluations se ramènent à la mesure d'effets moyens sur le groupe des individus traités et se répartissent en deux catégories selon que l'on se situe à l'échelle communale ou à l'échelle infra-communales :

À l'échelle communale, nous voulons connaitre l'influence de l'implantation de zonages environnementaux stricts sur des indicateurs de la dynamique urbaine (la croissance du nombre de logements de la commune, tous types de zones POS/PLU confondus, exprimée en taux) et agri- cole (l'évolution de la population agricole entre 1968 et 1999 et du nombre d'exploitations entre 1979 et 2000). Dans cette option, les individus statistiques sont les communes. Nous obtenons un pavage de l'espace en fonction des limites communales, avec un individu statistique par commune qui appartient soit au groupe des traités, soit au groupe des non traités (cf. carte 3 et annexe, cas DID1). Les communes sont considérées comme appartenant au groupe des traités lorsqu'elles accueillent un zonage environnemental strict dans leurs limites territoriales. Dans ce cadre, les estimations des effets visent à déterminer si les zonages environnementaux stricts limitent la dynamique urbaine et l'activité agricole des communes. Une réponse négative indiquerait que les zones constructibles existantes ou susceptibles de le devenir, permettent de dégager suffisamment d'espace pour maintenir la croissance urbaine et ne nuisent pas au maintien de l'activité agricole. Une réponse positive conforterait la position de certains maires

\section{Carte 3}

\section{Les groupes traités et non traités à l'échelle communale (DID1)}

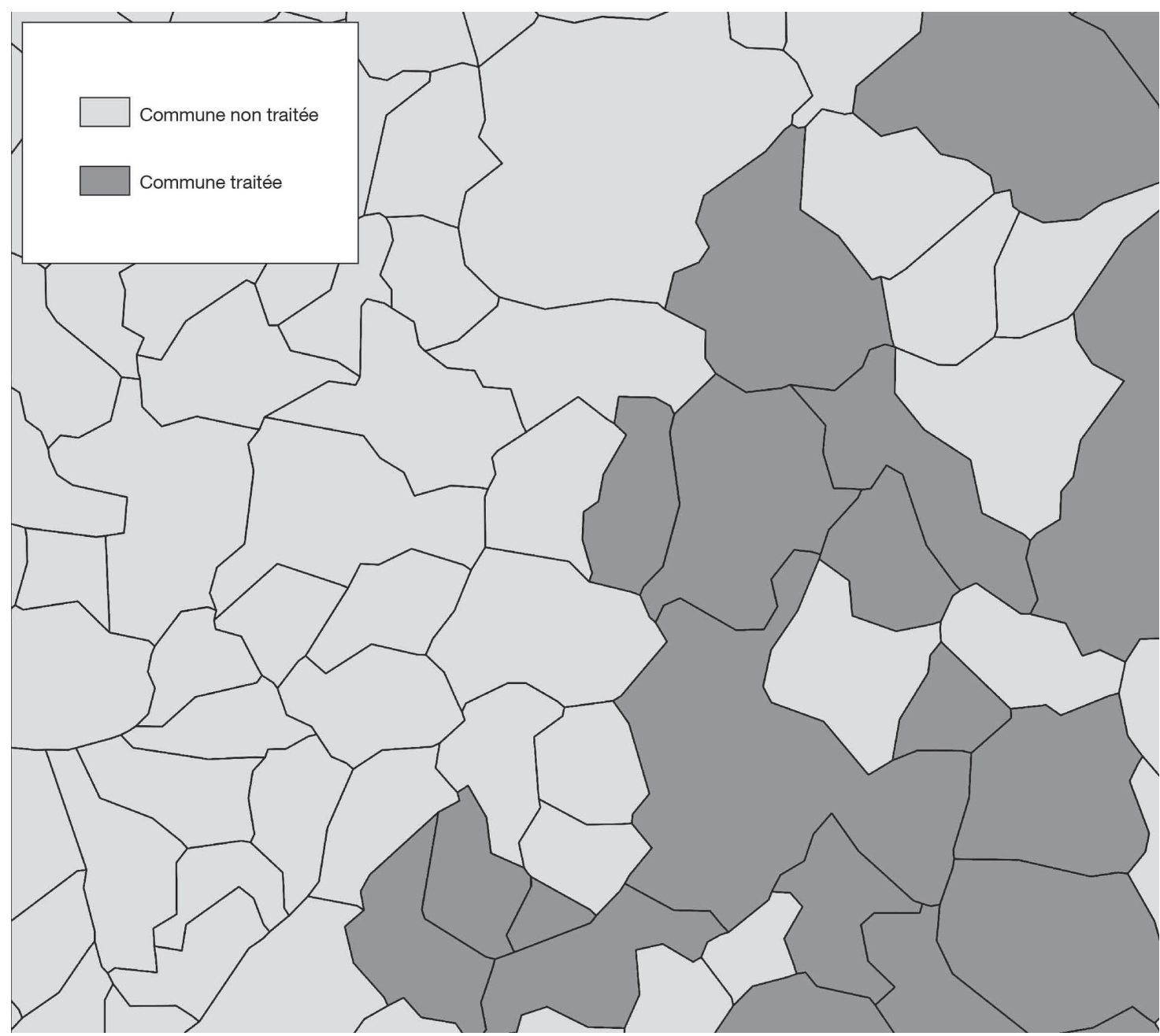

Sources : DREAL, MNHM, CRIGE PACA. 
jugeant les zonages environnementaux stricts pénalisant pour le développement urbain et/ou le maintien de l'activité agricole.

À l'échelle infra-communale, nous voulons savoir si les zonages environnementaux stricts créent des externalités locales dans leur voisinage immédiat et conduisent à des évolutions différentes des densités de logements au sein d'une même commune (exprimées en nombre de logements par hectare). À cette échelle on ne dispose pas de données géolocalisées sur l'agriculture antérieures aux années 1990. On limite donc l'analyse à celle des effets sur la densité résidentielle. Or, d'une part les densités s'organisent essentiellement en fonction du type de zonage d'urbanisme auquel la parcelle est rattachée et de la distance au noyau villageois (Dumas et al., 2005). D'autre part, les densités de logements varient beaucoup selon leur emplacement au sein d'une même commune : sur notre zone d'étude, on observe une densité médiane, exprimée en nombre de logements par hectare, de 7,9 pour les zonages de type U (habitat urbain dense), de 1,3 pour les zonages de type NA (habitat futur), de 1,7 pour les zonages de type NB (habitat diffus), de 0,17 pour les zonages de type NC (agriculture) et de 0,07 pour les zonages de type ND (milieux naturels). Il convient donc de tenir compte de cette première forme d'hétérogénéité dans le choix de la variable d'intérêt, par exemple en se focalisant sur un seul type de zonage. Les zones POS/PLU proches des zonages environnementaux sont aujourd'hui en très grande majorité des zones ND. On utilisera donc un pavage de la commune distinguant zones non traitées et zones traitées - définies comme les zones naturelles voisines d'un Zest - avec deux individus statistiques par commune traitée (cf. carte 4 et annexe, cas DID2).

Une fois le contour des zones tracé et leur classification réalisée, se posent trois questions

\section{Carte 4}

Les zones traitées (définies à partir d'une bande de $500 \mathrm{~m}$ autour des ZEST) et non traitées (définies comme le reste des zones ND) à l'échelle infra-communale (DID2)

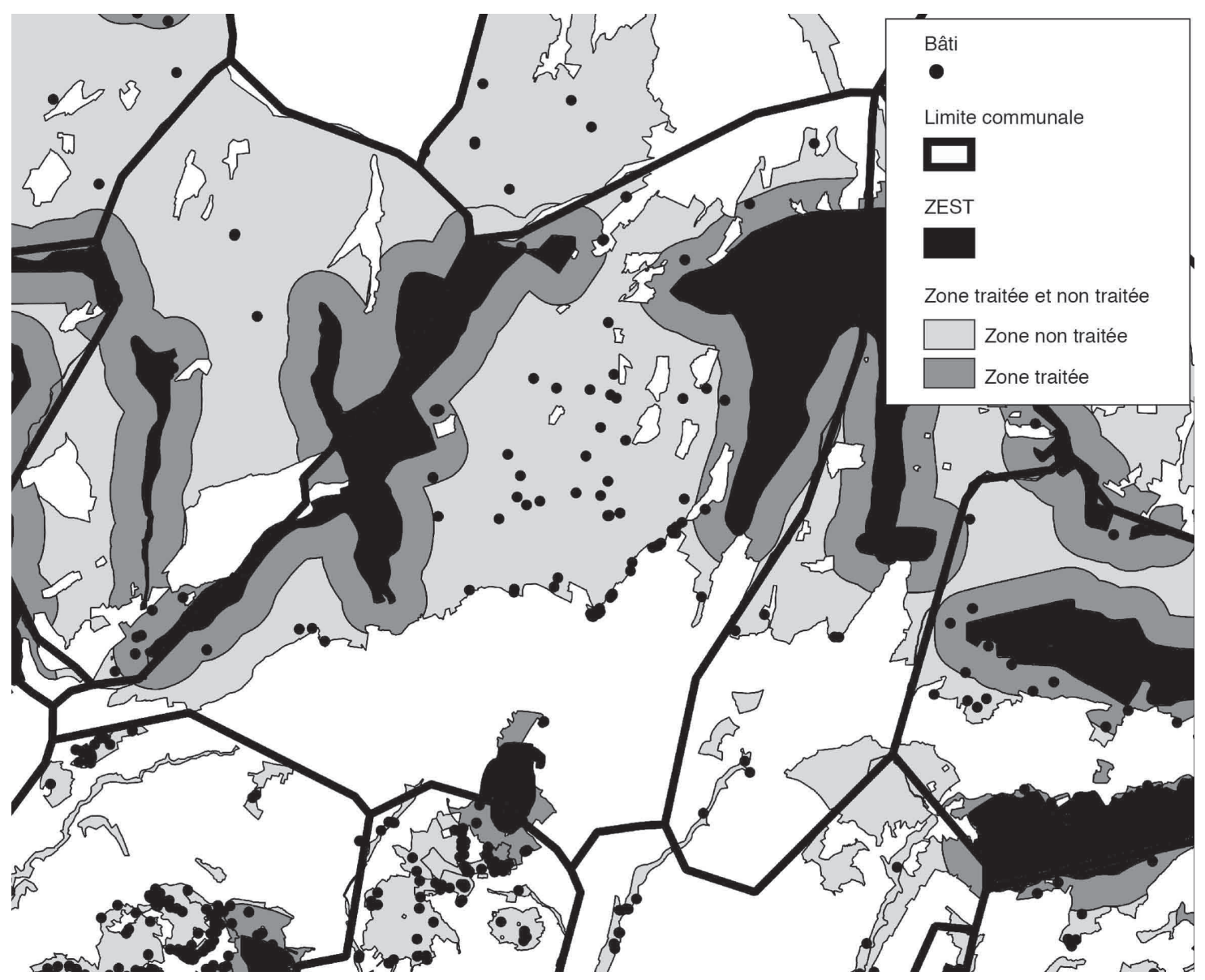

Lecture : les zones traitées (gris, nuance moyenne) sont définies comme les espaces en zone ND (gris clair) à moins de 500 mètres des limites des Zest (gris foncé) et situés à l'extérieur de ces dernières.

Sources : DREAL, MNHM, PCI, CRIGE PACA. 
importantes : la temporalité de la promulgation des zonages, la surface minimale de la zone traitée et la localisation des zones traitées et non traitées.

Tout d'abord, en ce qui concerne la chronologie, les zonages Zest ont pu être promulgués à des dates différentes. Il convient de ne retenir que les zonages dont les effets se manifestent au cours de la période d'étude. Pour ce faire, nous avons attribué à chaque zone une date minimale et une date maximale de promulgation à partir de l'ensemble des Zest situés dans la commune. Lorsqu'il y a des Zest avec des dates différentes (zonages qui évoluent dans le temps), il peut être délicat de fixer les dates pre et post traitement. On a choisi de se limiter aux 1300 zones ayant une date de promulgation unique entre 1975 et 1995 . Les effets pouvant ne se manifester que progressivement, en particulier lorsqu'ils consistent en construction d'habitations, nous avons fixé à 10 ans la période de latence minimum pour la date finale, c'est-à-dire 2005. Pour la date initiale, on ne retient pas de période de latence, et on prendra 1975. Cependant les recensements généraux de l'agriculture et de la population disponibles en 1975 ont été réalisés en 1968 et 1970. On mesure donc la différence de densité entre $1968 / 1970$ et 2005 , dans les communes ayant accueilli un Zest entre 1975 et 1995. Pour le nombre d'exploitations agricoles en fin

\section{Graphique I \\ Surface cumulée en Zest hors recouvrement (région Provence-Alpes-Côte d'Azur, surface en milliers d'hectares)}

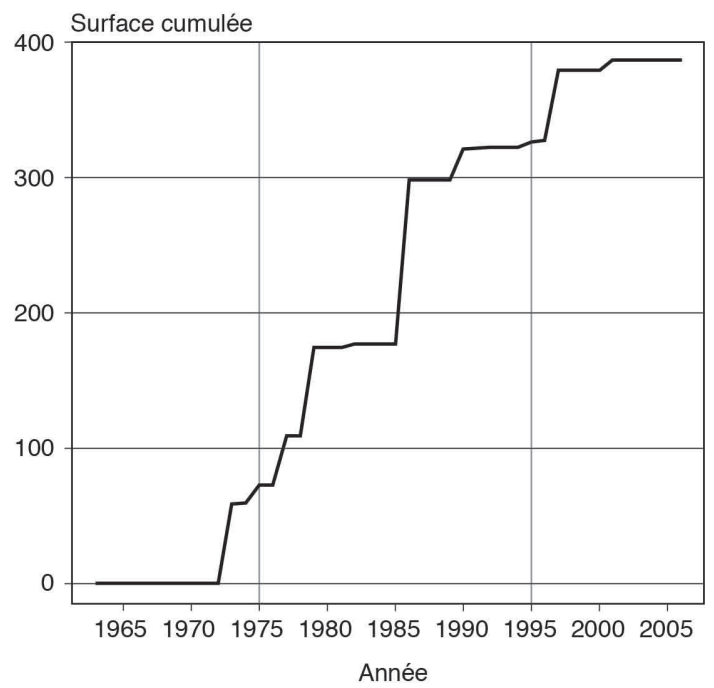

Lecture : en 1975, on observait 80000 ha environ couverts par au moins un zonage de type Zest. $70 \%$ des surfaces qui deviennent couvertes par au moins un zonage Zest le sont entre 1975 et 1995.

Sources : MNHM, DREAL. de période, on se réfère aux données du RGA 1979. Plus de $70 \%$ des surfaces hors recouvrement (7) en Zest sont comprises dans la période que nous avons retenue (cf. graphique I).

Ensuite, une zone traitée doit avoir une surface suffisante pour que la notion de densité y ait un sens et pour éviter une trop grande sensibilité de cette densité à une variation mineure de superficie. De plus, dans le cas d'une zone traitée définie comme une bande contiguë à la zest, un manque de profondeur expose au risque de surestimer les effets de débordements de la mesure. À l'inverse, une trop grande profondeur peut conduire à ne plus percevoir d'effet, par dilution ; voire même, pour de très petites communes, à une absence complète de zones non traitées. En conséquence, on a adopté pour largeur de la bande la distance minimale assurant une faible variance des densités de logements estimées. Pour cela, nous avons tiré une série d'échantillons aléatoires de 1000 localisations en zone ND (milieux naturels) dans un voisinage proche des Zest (moins de $5 \mathrm{~km}$ ), puis nous avons estimé l'évolution du nombre moyen de logements par hectare en fonction d'un éloignement à la Zest croissant par pas de 100 mètres : le choix du seuil a été fait graphiquement sur la base d'une trentaine de réplications du tirage aléatoire donnant des résultats similaires. On définit ce voisinage initial comme les espaces en zone ND à moins de 500 mètres des limites des Zest et situés à l'extérieur de ces dernières (cf. carte 4).

Enfin, si l'appariement entre les deux types de zones d'une même commune permet d'assurer aux groupes traités (définis à partir d'une bande de 500 mètres autour des Zest) et non traités (définis comme le reste des zones ND) un grand nombre de caractéristiques déterminantes communes dans le domaine foncier, ils peuvent cependant différer considérablement selon la distance aux zones construites (cf. carte 4). Or la distance au centre est un facteur déterminant des anticipations de modification des règles d'urbanisme et des prix fonciers (Geniaux et Napoléone, 2005 ; Geniaux et al., 2011). Sur l'ensemble des communes de la région de région Provence-Alpes-Côte d'Azur disposant de Zest, cette distance est en moyenne de 2887 mètres pour les zones non traitées et de 2237 pour les zones traitées - soit un écart de $30 \%$. On observe éga-

7. On entend par surface hors recouvrement, la somme des surfaces de chaque zonage moins la somme des surfaces de leur intersection. 
lement de fortes disparités infra-communales, avec une large majorité de communes où la distance moyenne est plus importante pour les zones traitées. Afin de construire des groupes de contrôle dont les distances moyennes au noyau villageois soient comparables, les zones ont été définies au moyen de deux critères : le critère de voisinage des Zest de $500 \mathrm{~m}$ de profondeur (pour la distinction entre individu traité et non traité, cf. supra) et la distance à l'urbanisation. On a utilisé pour cela une procédure géomatique visant à maintenir la continuité des zones et à éliminer le minimum de parcelles. Cette procédure consiste simplement à retirer à chacun des deux groupes, les parcelles les plus éloignées ou les plus proches du noyau urbain (8), et cela de façon itérative tant que l'écart entre les distances moyennes au centre des deux groupes traités et non traités est supérieur à $0,1 \%$. Cette procédure permet d'éliminer 19 communes dont les voisinages de Zest sont trop éloignés du noyau urbain relativement aux zones ND restantes : il n'était donc pas possible d'y construire deux groupes d'individus traités et non traités comparables.

Les méthodes d'appariement et les estimateurs ont été retenus en fonction de leur plus ou moins grande adaptation à l'échelon communal ou infra-communal, et aux modes de construction des groupes traités et non traités. Pour une présentation détaillée de ces méthodes, on se reportera à l'annexe.

\section{La densité de l'habitat est plus élevée et augmente plus vite dans les zones naturelles limitrophes aux Zest qu'à l'intérieur de ces dernières}

Dans la région Paca, la surface de Zest - hors propriétés du CELRL et avec recouvrement - est majoritairement composée des zones centrales de parc nationaux (58\%), suivies des réserves $(22 \%)$, des arrêtés de biotopes $(10 \%)$, des réserves naturelles $(8 \%)$, et des forêts soumises au régime ONF $(2 \%)$. Lorsque l'on procède à une estimation brute des parts d'emprises résidentielles (9) à l'intérieur des Zest et dans leur voisinage de $5 \mathrm{~km}, 10$ ans avant et 10 ans après leur promulgation, il apparait que les niveaux initiaux d'emprise résidentielle sont très faibles. Les zonages environnementaux de type Zest se caractérisent par une quasi-absence de construction ( $\mathrm{cf}$. tableau 1). Le niveau et la croissance des densités résidentielles des zones naturelles des POS/PLU alentours sont plus élevés (10 à 20 fois pour les niveaux de densités initiaux).

8. Au sein des classifications des types d'occupation du sol proposé par la couche Corine Land Cover de 2006, il existe une classe " 111 » correspondant au " tissu urbain continu » défini sur la base de critères de distance minimale entre pixels bâtis.

9. La part d'emprise résidentielle est la surface totale d'emprise au sol des bâtis divisée par la surface totale des parcelles.

\section{Tableau 1}

Densité l'intérieur des Zest et à dans la partie des zones naturelles ND située à moins de 5 km des Zest

\begin{tabular}{|c|c|c|c|}
\hline \multirow[b]{2}{*}{ Intérieur des zonages } & \multicolumn{2}{|c|}{ Densité de l'habitat } & \multirow{2}{*}{ Variation (en \%) } \\
\hline & À la date (t-10) & À la date $(t+10)$ & \\
\hline ONF (Office national des forêts) & 0,0004 & 0,0004 & 0 \\
\hline RNN (Réserve Naturelle Nationale) & 0,0008 & 0,0009 & 27 \\
\hline RBSZC (Réserve de biosphère, zone centrale) & 0,0014 & 0,0020 & 5 \\
\hline RAMSAR (convention Ramsar sur les zones humides) & 0,0080 & 0,0105 & 29 \\
\hline APB (Arrêté Préfectoral de Biotope) & 0,0014 & 0,0020 & 16 \\
\hline PNZC (Parc National, zone centrale) & 0,0004 & 0,0004 & 26 \\
\hline Voisinage à 5 km des zonages : & dens(t- 10) & $\operatorname{dens}(t+10)$ & $\Delta$ dens $\%$ \\
\hline ND proche ONF & 0,0160 & 0,0214 & 53 \\
\hline ND proche RNN & 0,0168 & 0,0236 & 47 \\
\hline ND proche RBSZC & 0,0333 & 0,0425 & 27 \\
\hline ND proche RAMSAR & 0,0158 & 0,0213 & 34 \\
\hline ND proche APB & 0,0286 & 0,0410 & 40 \\
\hline ND proche NPZC & 0,0037 & 0,0060 & 71 \\
\hline
\end{tabular}

Lecture : à l'intérieur des zonages ONF, la densité résidentielle, exprimée en nombre de logements par hectare, n'a pas augmenté entre les deux dates 10 ans avant et après la promulgation du zonage, alors que dans le voisinage des zonages ONF, elle passe de 0,016 habitation par hectare à 0,0214, ce qui représente une croissance de $53 \%$.

Sources : Insee, Agreste, DREAL, MNHM, CLC 2005, PERVAL, Safer. 


\section{Les trois variables caractérisant l'évolution des zones ont des distributions voisines sur le groupe traité et le groupe de contrôle initiaux}

Nous avons calculé les quintiles de la croissance du nombre de logements entre 1962 et 1968 (en taux), de l'emploi agricole en 1968 et du nombre de logements en 1968, sur le groupe traité et sur le groupe de contrôle initiaux, toutes observations confondues avant appariement (cf. tableau 2). Lorsque la répartition de chacun des deux groupes est proche de $20 \%$ sur l'ensemble des quintiles, alors les distributions peuvent être considérées comme similaires. On observe, avant appariement, que les trois variables ont chacune des distributions très proches sur chacun des deux groupes. Seuls trois quintiles affichent des différences supérieures à cinq points. L'emploi agricole est la seule variable pour laquelle on constate une différence significative entre les deux groupes avant appariement : elle ne satisfait pas, en effet, aux tests de comparaison (t-test et test de Wilcoxon).
En revanche, il existe des disparités entre les deux groupes en ce qui concerne la présence des différents types de zonages, dans la mesure où la présence d'un Zest implique presque toujours la présence de zonages de types Zeco ou Zesp. Par exemple, les Zesp représentent en moyenne $27,18 \%$ de la surface communale dans le groupe de contrôle contre $42,52 \%$ dans le groupe traité (cf. tableau 3) (10). Bien que les échantillons initiaux soient très comparables du point de vue de la dynamique urbaine ex ante et que celle-ci ne joue pas significativement dans la désignation d'un Zest (voir résultats du score de propension page suivante), nous utilisons la dynamique urbaine avant traitement comme variable d'appariement dans une variante de l'estimateur DID1 ; cette variable nous permet de contrôler les éventuels biais de sélection.

10. Nous n'utilisons pas pour autant la surface ou la présence de Zeco ou de Zesp comme variables d'appariement car cela nous permet de renforcer les conclusions que l'on tire de nos résultats.

Tableau 2

Distributions initiales de trois variables clés dans le groupe traité et le groupe de contrôle

\begin{tabular}{|c|c|c|c|c|c|c|c|c|}
\hline \multirow{2}{*}{ Variable } & \multirow{2}{*}{ Groupe } & \multirow{2}{*}{ t-test } & \multirow{2}{*}{$\begin{array}{l}\text { Wilcoxon } \\
\text { test }\end{array}$} & \multicolumn{5}{|c|}{ Percentile } \\
\hline & & & & $0-0,2$ & $0,2-0,4$ & $0,4-0,6$ & $0,6-0,8$ & $0,8-1$ \\
\hline \multirow{2}{*}{$\begin{array}{l}\text { Taux de croissance } \\
\text { du nombre de } \\
\text { logements } \\
1962-1968\end{array}$} & Traité & $t=0,5356$ & $W=76225$ & $\begin{array}{c}39 \\
(18,40 \%)\end{array}$ & $\begin{array}{c}36 \\
(16,98 \%)\end{array}$ & $\begin{array}{c}54 \\
(25,47 \%)\end{array}$ & $\begin{array}{c}41 \\
(19,34 \%)\end{array}$ & $\begin{array}{c}42 \\
(19,81 \%)\end{array}$ \\
\hline & Contrôle & $\begin{array}{c}p \text {-value }= \\
0,5926\end{array}$ & $\begin{array}{c}p \text {-value }= \\
0,4061\end{array}$ & $\begin{array}{c}142 \\
(20,49 \%)\end{array}$ & $\begin{array}{c}145 \\
(20,92 \%)\end{array}$ & $\begin{array}{c}145 \\
(22,37 \%)\end{array}$ & $\begin{array}{c}145 \\
(16,45 \%)\end{array}$ & $\begin{array}{c}137 \\
(19,77 \%)\end{array}$ \\
\hline \multirow{2}{*}{$\begin{array}{l}\text { Emploi agricole } \\
\text { en } 1968\end{array}$} & Traité & $t=2,0666$ & $W=87549$ & $\begin{array}{c}36 \\
(16,36 \%)\end{array}$ & $\begin{array}{c}45 \\
(20,45 \%)\end{array}$ & $\begin{array}{c}51 \\
(23,18 \%)\end{array}$ & $\begin{array}{c}38 \\
(17,27 \%)\end{array}$ & $\begin{array}{c}50 \\
(22,73 \%)\end{array}$ \\
\hline & Contrôle & $\begin{array}{c}p \text {-value = } \\
0,03982\end{array}$ & $\begin{array}{c}p \text {-value = } \\
0,04556\end{array}$ & $\begin{array}{c}167 \\
(22,85 \%)\end{array}$ & $\begin{array}{c}140 \\
(19,15 \%)\end{array}$ & $\begin{array}{c}145 \\
(19,84 \%)\end{array}$ & $\begin{array}{c}140 \\
(19,15 \%)\end{array}$ & $\begin{array}{c}139 \\
(19,02 \%)\end{array}$ \\
\hline \multirow{2}{*}{$\begin{array}{l}\text { Nombre de } \\
\text { logements } \\
\text { en } 1968\end{array}$} & Traité & $t=1,3696$ & $W=85560$ & $\begin{array}{c}34 \\
(15,25 \%)\end{array}$ & $\begin{array}{c}48 \\
(21,52 \%)\end{array}$ & $\begin{array}{c}53 \\
(23,77 \%)\end{array}$ & $\begin{array}{c}45 \\
(20,18 \%)\end{array}$ & $\begin{array}{c}43 \\
(19,28 \%)\end{array}$ \\
\hline & Contrôle & $\begin{array}{c}p \text {-value }= \\
0,1721\end{array}$ & $\begin{array}{c}p \text {-value }= \\
0,3189\end{array}$ & $\begin{array}{c}165 \\
(22,45 \%)\end{array}$ & $\begin{array}{c}146 \\
(19,86 \%)\end{array}$ & $\begin{array}{c}131 \\
(17,82 \%)\end{array}$ & $\begin{array}{c}145 \\
(19,73 \%)\end{array}$ & $\begin{array}{c}148 \\
(20,14 \%)\end{array}$ \\
\hline
\end{tabular}

Lecture : pour chaque percentile on a fait figurer la fréquence et, entre parenthèses, la proportion de la classe de percentile des variables taux de croissance du nombre de logements, emploi agricole, et nombre de logement dans les groupes traités et non traités (à l'échelle communale, avant traitement et avant appariement).

Sources : Insee, Agreste, DREAL, MNHM, CLC 2005.

Tableau 3

Répartition des types de zonages environnementaux avant appariement (Provence-Alpes-Côte d'Azur)

\begin{tabular}{|l|c|c|c|c|}
\hline \multicolumn{1}{|c|}{ Type de zonage } & Surface en $\mathrm{km}^{2}$ & $\begin{array}{c}\text { Part moyenne de la } \\
\text { surface communale } \\
\text { (en \%) }\end{array}$ & $\begin{array}{c}\text { Proportion de } \\
\text { communes } \\
\text { concernées (en \%) }\end{array}$ & $\begin{array}{c}\text { Part moyenne des } \\
\text { surfaces communales } \\
\text { (traité/contrôle) (en \%) }\end{array}$ \\
\hline Zest (hors CELRL) & 2670 & 8,40 & 23,00 & \\
\hline Zesp & 12113 & 38,14 & 82,00 & $42,52 / 27,18$ \\
\hline Zeco & 13758 & 43,33 & 94,70 & $56,30 / 15,94$ \\
\hline
\end{tabular}

Lecture : on observe $12113 \mathrm{~km}^{2}$ en zonage Zest en Paca représentant en moyenne 38,14 des surfaces totales des communes. I/s sont présents sur $82 \%$ des communes de Paca. À l'échelle infra-communale, ces zonages sont plus représentés des les zones traitées, avec en moyenne $42,52 \%$ contre $27,18 \%$ dans les zones de contrôle.

Sources : DREAL, MNHM, CLC 2005. 
À l'échelle infra-communale, lorsqu'on procède par appariement infra-communal (cas DID2) toutes les variables à l'échelle communale sont strictement égales. De plus, les Zesp et les Zeco représentent en moyenne des parts de la surface communale voisines dans les zones traitées et dans les zones de contrôle : ces moyennes sont de $46 \%$ contre $42 \%$ pour les Zesp et de $58 \%$ contre $52 \%$ pour les Zeco.

\section{Les Zest ne sont pas toujours localisées dans les zones les moins habitées}

L'estimation du score de propension sur la quasi totalité des communes de la région ProvenceAlpes-Côte d'Azur (858 communes sur 963) et pour une large gamme de variables candidates, rend compte de l'importance centrale des variables d'altitude et notamment de sa variance communale indiquant la présence de pentes (cf. tableau 4 et encadré 1). La relation à l'altitude moyenne est décroissante de 0 à environ $700 \mathrm{~m}$, puis croissante. Les Zest ont donc une probabilité plus importante de se situer sur le littoral ou en montagne. Les variables d'exposition (orientation moyenne des pentes) n'ont pas d'influence à cette échelle. On remarque également l'influence linéaire de la surface : cet effet est mécanique puisque plus la commune est grande, plus elle a de chance d'accueillir une Zest placée de façon aléatoire. La part de couverts forestiers et de zones en eaux joue également positivement. La densité résidentielle communale n'est pas, en revanche, un facteur significatif à cette échelle ; ce sont plutôt les populations communales lissées dans un rayon de $50 \mathrm{~km}$ qui influent sur la présence de Zest. On observe alors une relation croissante plutôt inattendue (pôles urbains exclus) qui indique que les zonages stricts ne sont pas toujours implantés dans les zones les moins habitées (11). Enfin, une part importante des effets imputables à des variables spatiales inobservables est capturée par le lissage spatial $(x, y)$ qui assure au total une qualité de prédiction correcte au modèle avec $90 \%$ de vrai 0 et $80 \%$ de vrai 1 (12).

Enfin, il existe une plage du score de propension sans support commun entre les deux groupes (approximativement de 0,9 à 1 ; cf. graphique II). Nous avons donc été conduits à sous échantillonner pour obtenir des estimations fiables (13).

11. Ces zones relativement peuplées sont en général littorales : le littoral est une zone résidentielle attractive ; c'est aussi une zone exposée à de nombreux dommages environnementaux, ce qui explique qu'il accueille fréquemment des Zest.

12. Lorsqu'on n'intègre pas les effets lissés de la longitude/latitude, le modèle n'explique alors plus que $20 \%$ de la variance.

13. Cette zone du PSM correspond aux communes de haute altitude et contiguës des parcs nationaux alpins.

Tableau 4

Estimation du score de propension

\begin{tabular}{|c|c|c|c|c|}
\hline Variables paramétriques & Coefficient & Écart-type & $z$ value & $p$-value \\
\hline Constante & $-5,400 e+00$ & $9,513 e-01$ & $-5,676$ & $1,38 \mathrm{e}-08^{\star \star \star}$ \\
\hline Surface communale & $1,274 \mathrm{e}-04$ & $5,510 e-05$ & 2,312 & 0,0208 * \\
\hline Proportion de couvert forestier & $3,265 e+00$ & $1,237 e+00$ & 2,639 & $0,0083^{* *}$ \\
\hline Nombre de logements en 1968 & $1,230 \mathrm{e}-04$ & $6,329 \mathrm{e}-05$ & 1,944 & 0,0519 \\
\hline Variables non paramétriques & Degré de liberté & $\begin{array}{c}\text { Degré de liberté } \\
\text { corrigé }\end{array}$ & Chi2 & p-value \\
\hline Écart type altitude & 4,021 & 4,521 & 24,27 & 0,000121 *** \\
\hline Coordonnées du barycentre $(\mathrm{x}, \mathrm{y})$ & 26,954 & 27,454 & 130,72 & $2,11 \mathrm{e}-15^{\star \star \star}$ \\
\hline Altitude moyenne & 8,113 & 8,613 & 22,30 & 0,006376 ** \\
\hline $\begin{array}{l}\text { Montant global des mutations } \\
\text { foncières }\end{array}$ & 5,646 & 6,146 & 13,94 & 0,033074 * \\
\hline $\begin{array}{l}\text { Population } 1999 \text { sur } 50 \text { km, } \\
\text { lissée spatiallement }\end{array}$ & 6,856 & 7,356 & 19,09 & $0,009857^{* *}$ \\
\hline Proportion de zones en eaux & 2,370 & 2,870 & 11,55 & $0,008027^{* *}$ \\
\hline \multicolumn{2}{|l|}{$\mathrm{R} 2(\operatorname{adj})=0,578$} & Obs/préd & VRAI & FAUX \\
\hline \multirow{2}{*}{\multicolumn{2}{|c|}{$\begin{array}{l}\text { Variance expliquée }=56,5 \% \\
N=858\end{array}$}} & 0 & 629 & 26 \\
\hline & & 1 & 57 & 146 \\
\hline
\end{tabular}

Lecture : on utilise un modèle logit par GAM Géoadditif. La proportion de couvert forestier communal a un impact positif (coefficient positif de 1,274e-04) significatif au seuil de $5 \%$ ( $p$-value inférieure à 0,05 ) sur la probabilité pour la commune d'être couvert par un zonage environnemental strict (Zest). Le modèle permet d'obtenir un pseudo R2 de 0,578 avec 57 fausses prédictions de traitement, 26 fausses prédictions de non traitement, et un total de 775 prédictions correctes.

Sources : Insee, Agreste, DREAL, MNHM, CLC 2005, PERVAL, Safer. 


\section{La construction a été sensiblement freinée au voisinage immédiat des zonages environnementaux stricts}

À l'échelle communale (DID1), les effets propres des Zest s'avèrent significatifs (14). Ils sont positifs sur les logements et négligeables sur la population agricole et le nombre d'exploitations en activité dans la commune (cf. tableau 5).

Concernant l'évolution du nombre de logements entre 1968 et 2005, les résultats montrent un effet positif significatif, de l'ordre de $20 \%$, pour les deux estimateurs en appariement par plus proche score de propension bornés par caliper (15) et sans remise. Les estimations de l'effet moyen des Zest sur les communes traitées sont en effet très proches dès lors que l'on contrôle la qualité des appariements à la fois sur les premiers et seconds moments des covariables $X$ ( $t$ balancing test et $K S$ balancing test). Nous avons retenu comme référence l'évaluation en plus proche voisin avec un seuil de caliper à 0,05, ainsi qu'un appariement en fonction du Propensity Score et de la variable d'intérêt avant promulgation du zonage (DID1). L'utilisation d'un seuil de caliper conduit à ne considérer qu'environ $65 \%$ des observations traitées dans les calculs (16).
En ce qui concerne l'évolution de la population agricole entre 1968 et 1999, aucun effet significatif n'est repérable : le déclin de la population agricole et du nombre d'exploitations a été comparable dans les deux groupes de communes. Le recul de la population agricole de ProvenceAlpes -Côte d'Azur entre 1968 et 1999 a été de -70794 actifs agricoles (- $60 \%$ ) pour l'ensemble des communes.

Dans les communes où une zone environnementale stricte a été promulguée, la croissance du nombre de logements a donc été plus soutenue que dans les communes similaires n'ayant pas accueillies ce type de zone (20\% en moyenne), sans pour autant nuire à l'activité agricole (effet non significatif). On peut alors se demander si l'effet est uniformément réparti sur le territoire de la commune, ou s'il est plus important à proximité des zonages

\footnotetext{
14. Les variances conditionnelles des estimateurs sont estimées à partir des propositions de Abadie et Imbens (2006).

15. Le caliper permet de donner la valeur maximum entre scores de propension au-delà de laquelle il ne peut plus y'avoir d'appariement.

16. Avec un lissage par noyau (Kernel Matching), les appariements conduisent à de très nettes différences de distribution des covariables entre groupe traité et groupe non traité, qui invitent à ne pas tenir compte des résultats estimés avec ce type d'es-
} timateur.

\section{Graphique II}

Densité de probabilité du score de propension dans chacun des groupes

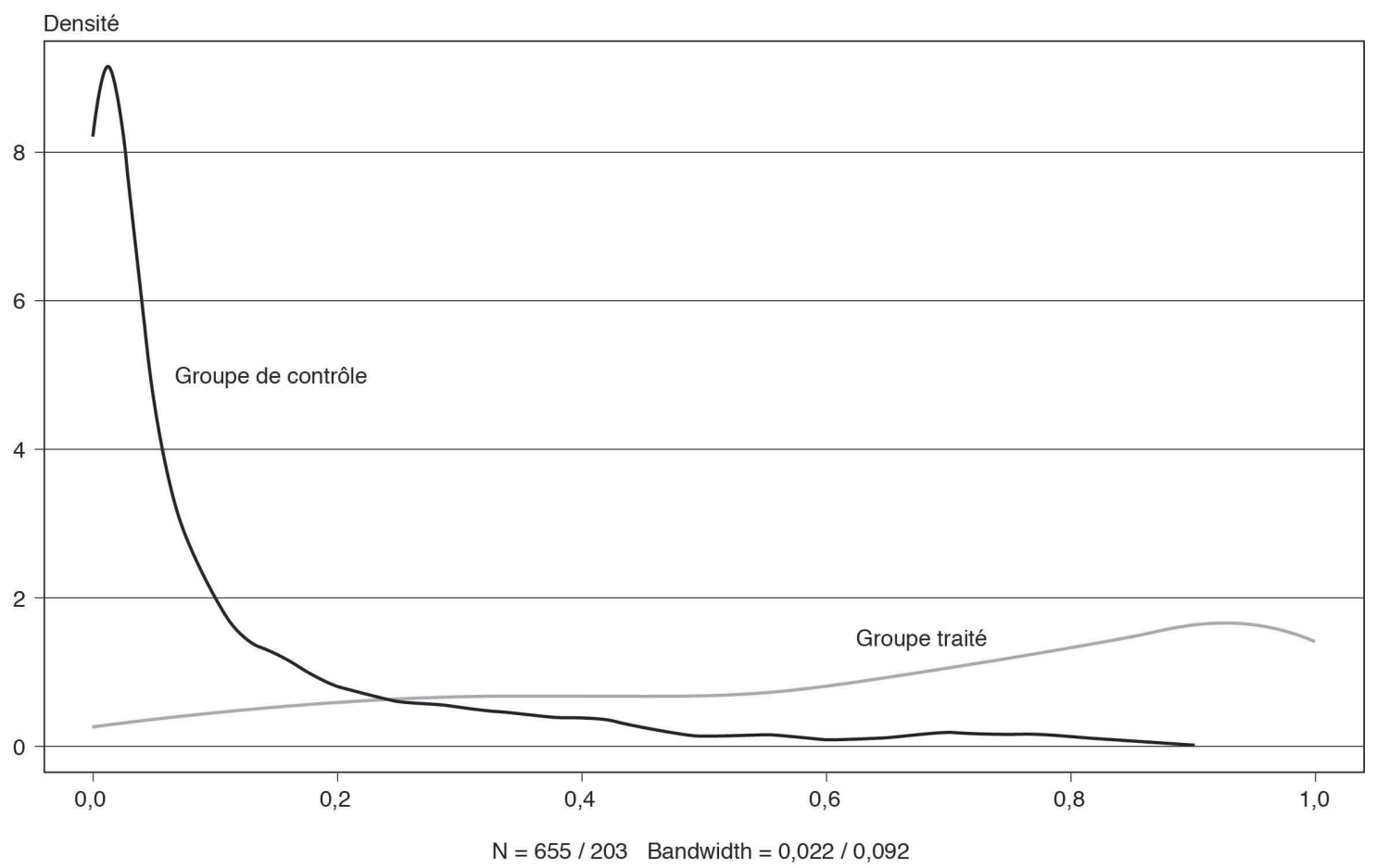

Lecture : l'observation des densités lissées du score de propension dans chacun des groupes permet de voir que pour une valeur supérieure à 0,9 , il n'y a aucun individu statistique dans le groupe de contrôle.

Sources: Insee, Agreste, DREAL, MNHM, CLC 2005, PERVAL, Safer. 
Tableau 5

Estimation des effets propres des Zest, option DID1

\begin{tabular}{|c|c|c|c|c|c|c|c|c|c|c|c|}
\hline \multirow{2}{*}{\multicolumn{2}{|c|}{ DID1 }} & \multicolumn{4}{|c|}{ Échantillon } & \multirow{2}{*}{\multicolumn{2}{|c|}{$\begin{array}{c}\text { Évolution du } \\
\text { nombre de } \\
\text { logements entre } \\
1968 \text { et } 2005 \\
\text { Estimateur }\end{array}$}} & \multirow{2}{*}{\multicolumn{2}{|c|}{ 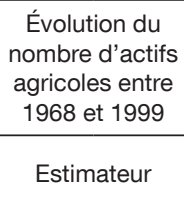 }} & \multirow{2}{*}{\multicolumn{2}{|c|}{ 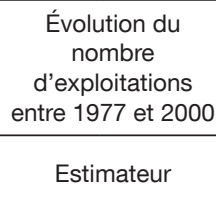 }} \\
\hline & & \multirow{3}{*}{$\begin{array}{c}\text { Traité } \\
\mathrm{n} 1 \\
7 ?\end{array}$} & \multirow{2}{*}{$\begin{array}{c}\text { Non } \\
\text { Traité }\end{array}$} & \multicolumn{2}{|c|}{$\begin{array}{c}\text { Échec des } \\
\text { banlancing test }\end{array}$} & & & & & & \\
\hline \multirow{4}{*}{$\begin{array}{l}\text { Plus proche } \\
\text { voisin avec } \\
\text { caliper } \\
\text { DID1a }\end{array}$} & caliper & & & $\mathrm{T}$ test & $\mathrm{KS}$ test & DID & $\mathrm{t}$ & DID & $\mathrm{t}$ & DID & $\mathrm{t}$ \\
\hline & 0,01 & & 72 & 0 & 0 & 0,23 & $2,21^{\star}$ & 0,16 & 1,31 & $-0,02$ & $-0,35$ \\
\hline & 0,05 & 128 & 128 & 0 & 0 & 0,16 & $2,03^{\star}$ & $-0,2$ & $-0,88$ & $-0,01$ & $-0,31$ \\
\hline & 0,2 & 141 & 141 & 0 & 1 & 0,22 & $2,52^{\star \star}$ & $-0,17$ & $-0,79$ & $-0,05$ & $-1,59$ \\
\hline \multirow{3}{*}{$\begin{array}{l}\text { Plus proche } \\
\text { voisin avec } \\
\text { support } \\
\text { commun }\end{array}$} & Stratifié & $\mathrm{n} 1$ & $\mathrm{n} 2$ & $\mathrm{~T}$ test & KS test & DID & $\mathrm{t}$ & DID & $\mathrm{t}$ & DID & \\
\hline & DID1b & 117 & 117 & 1 & 2 & 0.17 & $2.17^{\star}$ & $-0,08$ & -0.86 & $-0,03$ & $-0,82$ \\
\hline & $\begin{array}{c}\text { DID1c } \\
\text { Mahalanobis }\end{array}$ & 128 & 128 & 1 & 1 & 0.18 & $2.19^{\star}$ & $-0,11$ & $-1,21$ & -0.03 & -1 \\
\hline \multirow{4}{*}{$\begin{array}{l}\text { Noyau } \\
\text { gaussien } \\
\text { DID1d }\end{array}$} & Bandwidth & $\mathrm{n} 1$ & $\mathrm{n} 2$ & $\mathrm{~T}$ test & KS test & DID & $\mathrm{t}$ & DID & $\mathrm{t}$ & DID & $\mathrm{t}$ \\
\hline & 0,01 & 128 & 576 & 1 & 7 & 0,251 & $2,73^{\star *}$ & 0,04 & 0,41 & 0,01 & 0,41 \\
\hline & 0,05 & 128 & 576 & 1 & 7 & 0,173 & 1,88 & 0,05 & 1,06 & 0,01 & 0,27 \\
\hline & 0,2 & 128 & 576 & 0 & 7 & 0,07 & 0,79 & 0,04 & 0,75 & 0,01 & 0,29 \\
\hline
\end{tabular}

Lecture : l'effet propre de la présence d'un zonage environnemental (Zest) dans une commune est de 0,23 (soit 23 \%) sur le taux de croissance des logements entre 1968 et 2005, lorsqu'on utilise un estimateur en double différence avec appariement par plus proche PSM (propensity score matching) sans remise avec un seuil de caliper de 0,01 (NN caliper).

Sources : Insee, Agreste, DREAL, MNHM, CLC 2005, PERVAL, Safer.

Tableau 6

Estimation des effets propres des Zest, option DID2

\begin{tabular}{|c|c|c|c|c|c|}
\hline \multirow{2}{*}{ DID2 $\mathrm{k}=500 \mathrm{~m}$} & \multicolumn{2}{|c|}{ Échantillon } & \multicolumn{3}{|c|}{$\begin{array}{c}\text { Évolution de la densité résidentielle en zone ND } \\
\text { entre } 1970 \text { et } 2005\end{array}$} \\
\hline & Traité & Non Traité & & teur & $\begin{array}{l}\text { Échec des } \\
\text { banlancing test }\end{array}$ \\
\hline \multirow{2}{*}{$\begin{array}{l}\text { Plus proche voisin avec appariement } \\
\text { exact }\end{array}$} & $\mathrm{n} 1$ & $\mathrm{n} 2$ & DID & $\mathrm{t}$ & T test \\
\hline & 92 & 92 & $-0,143$ & $-4,579^{\star \star \star *}$ & 0 \\
\hline
\end{tabular}

Lecture : l'effet propre d'avoir un zonage environnemental (Zest) à moins de $500 \mathrm{~m}$ en zone ND est de - 0,21 (soit - 21 \%) sur la densité résidentielle entre 1970 et 2005, lorsqu'on utilise un estimateur en double différence avec appariement exact sur le PSM (propensity score matching qui est invariant à l'échelle infra-communale).

Sources : Insee, Agreste, DREAL, MNHM, CLC 2005, PERVAL, Safer.

environnementaux. Les estimateurs $D I D 2_{k}$ permettent de répondre en partie cette question. Nous avons estimé les effets sur les densités en ne considérant que les zones naturelles des documents d'urbanisme (ND des POS et $\mathrm{N}$ des PLU) et en privilégiant des appariement intra-communaux entre zones proches et zones éloignées des zonages environnementaux. L'effet est négatif et significatif : - $14 \%$ pour la «zone tampon » de 0 à 500 mètres définie plus haut (cf. tableau 6). Les Zest induiraient donc une croissance plus faible à leur proximité et exercerait une influence « protectrice » effective.

Il faut toutefois garder à l'esprit que l'hétérogénéité des dates de promulgation des zonages environnementaux nous a conduit à estimer la différence de densité entre les années 1970 et 2005, en ne considérant que les zonages environnementaux promulgués entre 1975 et 1995. Des estimations des effets prenant en compte la date effective de chaque zonage et s'appuyant sur des estimations de densité à date flottante permettraient d'estimer un effet propre exprimé sous forme de taux de croissance annuel moyen. Une autre voie d'amélioration possible consiste à se placer dans un cadre de traitement continu (Imbens et Hirano, 2004) en fonction de la distance aux zonages environnementaux. Une telle méthode permettrait de connaître avec plus de précision la distribution spatiale de ces effets. Enfin, un prolongement naturel de cette étude consisterait à de tester l'existence de tels effets dans des régions où la pression foncière et la densité des zonages environnementaux sont moins importantes qu'en région Provence-Alpes-Côte d'Azur. 


\section{BIBLIOGRAPHIE}

Abadie A. (2005), « Semiparametric differencein-differences estimators », Review Of Economic Studies, vol. 72, n 1, pp. 1-19.

Abadie A. et Imbens G. (2006), « Large Sample Properties of Matching Estimators for Average Treatment Effects », Econometrica, vol. 74, n 1 , pp. 235-267.

Abadie A. et Imbens G. (2008), « On the Failure of the Bootstrap for Matching Estimators », Econometrica, vol. 76, n 6, pp. 1537-1557.

Anderson K. et Weinhold D. (2008), « Valuing future development rights : The costs of conservation easements », Ecological Economics, vol. 68, pp. 437-446.

Ashenfelter O. (1978), « Estimating the Effects of Training Programs on Earnings ", Review of Economics and Statistics, vol. 60, pp. 47-57.

Cochran W.G. et Rubin D.B. (1973), «Controlling Bias in Observational Studies : A Review », Sankhya, Series A 35, pp. 417-446.

Deverre C. et de Sainte Marie C. (2008), « L'écologisation de la politique agricole européenne. Verdissement ou refondation des systèmes agro-alimentaires ? ", Revue d'Études en Agriculture et Environnement, vol. 89, $\mathrm{n}^{\circ}$ 4, pp. 83-104.

Dumas E., Geniaux G., Napoléone C., Bartoli C. et Cezanne-Bert P. (2005), Identification qualitative des espaces disponibles pour l'urbanisation nouvelle, Rapport d'étude CESSA/INRA pour le Conseil régional Provence Alpes Côte d'Azur CESSA, 280 pages.

Ellickson R. (1977), « Suburban growth controls : an economic and legal analysis ", Yale Law Journal, vol. 86, n 3, pp. 385-511.

Evans A.W. (1999), « The land market and government intervention », dans Handbook of regional and Urban Economics (Mills and Chesire eds), Elsevier.

Fischel W. (1985), The economics of zoning law, Baltimore MD : John Hopkins University Press.

Fougère D. (2010), « les méthodes économétriques d'évaluation », Revue Française des Affaires sociales, $\mathrm{n}^{\circ} 1-2$, pp. 105-128.
Geniaux G. et Napoléone C. (2005), « Rente foncière et anticipations dans le périurbain ", Économie et Prévision, ${ }^{\circ}$ 168, pp. 77-95.

Geniaux G. et Napoléone C. (2008), « Semiparametric tools for spatial hedonic models : an introduction to mixed geographically weighted regression and geoadditive models » In Baranzini, A., Ramirez, J., Schaerer, C., and Thalmann, P., editors, Hedonic Methods in Housing Markets Pricing Environmental Amenities and Segregation, pp. 101-127, New York : Springer.

Geniaux G., Ay J.-S. et Napoléone C. (2011). «A spatial hedonic approach on land use change anticipations ", Journal of Regional Science, vol. 51, $\mathrm{n}^{\circ}$ 5, pp. 967-986.

Geniaux G., Pluvinet P., Cezanne-Bert P. et Jouve J. (2009), « Identification qualitative des espaces disponibles pour une urbanisation nouvelle. Test sur 3 SCOT - Simulations à moyen-long terme : méthodologie ", Rapport d'étude CESSA/ INRA pour la DRE PACA, 41 pages.

Hastie T. et Tibshirani R. (1990), Generalized additive models, Chapman and Hall.

Heckman J., Ichimura H. et Todd P. (1998), « Matching as an Econometric Evaluation Estimator », Review of Economic Studies, $\mathrm{n}^{\circ}$ 65, pp. 261-294.

Imbens G. et Hirano K. (2004), « The Propensity score with continuous treatment ", chapter for Missing data and Bayesian Method in Practice : Contributions by Donald Rubin Statistical Family.

Imbens G. et Wooldridge J.M. (2009), « Recent Developments in the Econometrics of Program Evaluation », Journal of Economic Literature, vol. $47, \mathrm{n}^{\circ} 1$, pp. 5-86.

Jaeger K.W. et Plantinga A.J. (2007), « How Have Land-use Regulations Affected Property Values in Oregon? », Technical report of Oregon State University, Special Report 1077.

Lynch L., Gray W. et Geoghegan J. (2007), «Are farmland preservation program easement restrictions capitalized into farmland prices ? What can a propensity score matching analysis tell us ? ", Review Of Agricultural Economics, vol. 29, $\mathrm{n}^{\circ} 3$, pp. 502-509. 
Lynch L. et Liu X.P. (2007), « Impact of designated preservation areas on rate of preservation and rate of conversion : Preliminary évidence », Proceedings Paper Conference of Annual Meeting of the American-Agricultural-EconomicsAssociation, Portland, Oregon.

McDonald J.F. et McMillen D.P. (2004), «Determinants of suburban development controls : a fischel expédition », Urban Stuties, vol. 41, $\mathrm{n}^{\circ} 2$, pp. 341-361.

McMillen D.P. et McDonald J.F. (2002), "Land values in a newly zoned city », Review Of Economics And Statistics, vol. 84, $\mathrm{n}^{\circ} 1$, pp. 62-72.

Netusil N.R. (2005), " The effect of environmental zoning and amenities on property values : Portland, Oregon "), Land Economics, vol. 81, $\mathrm{n}^{\circ} 2$, pp. 227-246.

Rubin D.B. (1978), « Bayesian inference for causal effects: The Role of Randomization », Annals of Statistics, vol. 6, $\mathrm{n}^{\circ}$ 1, pp. 34-58.
Sekhon J.S. (2007), « Multivariate and Propensity Score MatchingSoftware with Automated Balance Optimization : The Matching package for R. ", Journal of Statistical Software, vol. 10, $\mathrm{n}^{\circ} 2$, pp. 1-51.

Stigler G. (1971), " The theory of economic régulation ", Bell Journal of Economics and Management Science, vol. 2, n 1, pp. 1-21.

Towe C. et Bockstael. N.E. (2005), « Testing the Effect of Neighboring Open Space on Development Using a Real Options Model. », Paper presented at the INRA Workshop on the Role of Open Space Amenities and Green Amenities in Residential Decentralization, Dijon, France.

Wood S. (2006), Generalized Additive Models : An introduction with $R$, Chapman \& Hall/CRC, 391 pages.

Zhou J., McMillen D.P. et McDonald J.F. (2008), « Land values and the 1957 Comprehensive Amendment to the Chicago Zoning Ordinance ", Urban Studies, vol. 45, n 8, pp. 1647-1661. 


\section{MODE D'APPARIEMENT ET ESTIMATEURS}

On a retenu les méthodes d'appariement et les estimateurs les plus adaptés à l'échelon communal ou infra- communal, et aux modes de construction des groupes traités et non traités (cf. tableau ci-dessous).

Tableau de synthèse des options méthodologiques

\begin{tabular}{|c|l|l|l|c|}
\hline Échelle & \multicolumn{1}{|c|}{$\begin{array}{l}\text { Variable } \\
\text { d'intérêt }\end{array}$} & Distinction traités/non traités & \multicolumn{1}{c|}{$\begin{array}{c}\text { Appariement de } \\
\text { l'estimateur }\end{array}$} \\
\hline \multirow{5}{*}{ Commune } & $\begin{array}{l}\text { Nombre de } \\
\text { logements; } \\
\text { Population } \\
\text { Agricole }\end{array}$ & $\begin{array}{l}\text { Existence ou pas d'un Zest } \\
\text { sur la commune }\end{array}$ & $\begin{array}{l}\text { Dar plus proche voisin avec caliper sur score de } \\
\text { propension. } \\
\text { - Par plus proche voisin avec support commun } \\
\text { sur score de propension (à l'intérieur de 9 strates } \\
\text { définit par le logement et sa dynamique avant } \\
\text { traitement). } \\
\text { - Par plus proche voisin avec support commun } \\
\text { sur score de propension et taux de croissance } \\
\text { des logements avant traitement (distance de } \\
\text { mahalanobis). } \\
\text { - Par noyau sur score de propension. }\end{array}$ \\
\hline Zones ND & $\begin{array}{l}\text { Densité de } \\
\text { logements } \\
\text { des zones ND }\end{array}$ & $\begin{array}{l}\text { Distance au Zest inférieur } \\
\text { ou pas à 500 mètres, sous } \\
\text { contrainte d'égalité des distan- } \\
\text { ces moyennes au noyau urbain }\end{array}$ & DiD1c \\
Appartenance à une même commune & DID1d \\
\hline
\end{tabular}

\section{Échelon communal (cas DID1)}

On s'appuie sur deux modes d'appariement différents : par plus proche voisin (NN pour Nearest Neighbour) ou par noyau (KM pour Kernel Matching).

L'appariement par plus proche voisin permet de comparer les groupes de communes dont les caractéristiques sont les plus proches. II repose sur un seuil de différence maximal entre les valeurs de la variable de contrôle et son contrefactuel (caliper matching, Cochran et Rubin, 1973). Cette méthode permet de contrôler la qualité des appariements et, par là même, de traiter la question de support commun entre groupe traité et non traité. Si l'on note $Y$ la différence de résultat de l'observation $i$ pour la période, $N_{1}$ le nombre d'individus du groupe traité $I_{1}$, $I_{0}$ le groupe des non traités, $P(x)$ le score de propension de l'individu $j$, et $x$ le vecteur de variable jouant sur la probabilité de traitement, alors ce premier estimateur par plus proche voisin s'écrit :

$$
\Delta^{T T}=\frac{1}{N_{1}} \sum_{i \in I_{1}}\left\{\Delta Y_{i}-\Delta Y_{\tilde{j}(i)}\right\}(1)
$$

Où $\tilde{j}(i)=\operatorname{argmin}_{j \in I_{0}}\left\|X_{i}-X_{j}\right\|_{\Sigma^{-1}}$ est l'observation minimisant les différences de caractéristiques $X$ entre $i$ et son contrefactuel non traité.

L'appariement par noyau considère une moyenne pondérée des observations des $n$ voisins les plus proches de $i$ plutôt que le seul plus proche voisin. Cet estimateur pondère les observations de l'échantillon de contrôle associées à chaque observation traitée $i$, en accordant un poids plus élevé aux observations dont la caractéristique désirée (généralement le score de propension) est proche de celle l'observation traitée $i$. Le schéma de pondération est défini à partir d'un seuil appelé bandwidth : plus ce seuil est faible, plus on réduit le poids des observations ayant des caractéristiques éloignées. Dans notre étude, nous utilisons les poids de $K 0$ définis à partir d'un noyau de type Gaussien et trois valeurs de bandwidth allant de 0,01, 0,05 et 0,2. Proposé par Heckman et al. (1998), cet estimateur par noyau s'écrit :

$$
\begin{aligned}
& \Delta^{T T}=\frac{1}{N_{1}} \sum_{i \in I_{1}} \\
& \left\{\Delta Y_{i}-\sum_{j \in I_{0}} \frac{K\left(\left(p\left(z_{j}\right)-p\left(z_{i}\right)\right) / h\right)}{\sum_{j \in I_{0}} K\left(\left(p\left(z_{j}\right)-p\left(z_{i}\right)\right) / h\right)} \Delta Y_{j}\right\}
\end{aligned}
$$

Les appariements en fonction du score de propension peuvent se révéler insuffisants s'il y a une grande variabilité des situations des individus statistiques concernant la variable d'intérêt avant traitement. On sait par exemple que l'évolution des densités et du nombre de logements dans le temps n'est pas linéaire et que les niveaux de croissance annuels de ces deux variables sont très dépendants de leur niveau initial en $t_{0}$. La courbe peut être, selon le type de zonage d'urbanisme et la valeur initiale, convexe puis concave ou uniquement concave. Comme le mentionnent Imbens et Wooldridge (2009), il convient alors de tenir compte de cette hétérogénéité dans les variables d'appariement (appariement en fonction de la variable d'intérêt retardée ou de sa dynamique avant traitement), complémentairement au score de propension. Pour cette raison, toutes nos estimations comparent des taux de croissance et non des variables en niveau. Pour ce qui est de la dynamique avant traitement, nous avons testé différentes variantes possibles (notamment l'usage de distance de Mahalanobis (1) sur le score de propension et les variables renseignant la dynamique avant traitement). Nous avons retenu cinq percentiles sur le taux de croissance du nombre de logements entre 1962 et 1968. Dans la version DID1b (cf. tableau ci-dessus) on utilise un appariement par plus proche score de propension à l'intérieur des percentiles et un appariement par distance de Mahalanobis dans la version DID1c. On peut remarquer que les répartitions entre communes traitées et non traitées dans ces percentiles sont équilibrées et que les Zest n'apparaissent pas forcément dans des communes où la dynamique

1. La distance de Mahalanobis est une mesure de similarité entre séries de données. 
d'urbanisation est particulière (faible par opportunité, ou forte par réaction) (cf. tableau 2 du texte).

Échelon infra-communal (cas DID2)

L'appariement entre observations traitées et non traitées se fait sur la base de l'appartenance à une même commune. Ce mode d'appariement présente l'avantage de garantir des observations similaires en termes de pression foncière, de caractéristiques de couverts et de types de zonages et incidemment de probabilité de recevoir un zonage environnemental strict. Notre estimation est basée sur les groupes traités et non traités, sous contrainte d'égalité des distances moyennes au noyau urbain (DID2). Nous avons privilégié les estimations des variances conditionnelles des estimateurs (1) et (2) asymptotiques sur la base des propositions de Abadie et Imbens (2006), dont Abadie et Imbens (2008) démontrent la validité pour ce type d'estimateur, même sur petits échantillons. 
